\title{
Automatic Selection of DBS Target Points Using Multiple Electrophysiological Atlases
}

\author{
Pierre-Francois D’Haese ${ }^{1,2}$, Srivatsan Pallavaram ${ }^{1}$, Ken Niermann ${ }^{4}$, John Spooner ${ }^{3}$, \\ Chris Kao $^{3,5}$, Peter E. Konrad ${ }^{3}$, and Benoit M. Dawant ${ }^{1}$ \\ ${ }^{1}$ Department of Electrical Engineering and Computer Science, \\ Vanderbilt University, Nashville, TN, USA \\ ${ }^{2}$ Université Catholique de Louvain, Louvain-la-Neuve, Belgique \\ ${ }^{3}$ Departments of Neurosurgery, Vanderbilt Medical Center, Nashville, TN, USA \\ ${ }^{4}$ Departments of Radiology, Vanderbilt Medical Center, Nashville, TN, USA \\ ${ }^{5}$ Sentient Medical Systems, Cockeysville, MD 21030
}

\begin{abstract}
In this paper we study and evaluate the influence of the choice of a particular reference volume as the electrophysiological atlas on the accuracy of the automatic predictions of optimal points for deep brain stimulator (DBS) implants. We refer to an electrophysiological atlas as a spatial map of electrophysiological information such as micro electrode recordings (MER), stimulation parameters, final implants positions, etc., which are acquired for each patient and then mapped onto a single reference volume using registration algorithms. An atlas-based prediction of the optimal point for a DBS surgery is made by registering a patient's image volume to that reference volume, that is, by computing a correct coordinate mapping between the two; and then by projecting the optimal point from the atlas to the patient using the transformation from the registration algorithm. Different atlases, as well as different parameterizations of the registration algorithm, lead to different and somewhat independent atlas-based predictions. We show how the use of multiple reference volumes can improve the accuracy of prediction by combining the predictions from the multiple reference volumes weighted by the accuracy of the non-rigid registration between each of the corresponding atlases and the patient volume.
\end{abstract}

\section{Introduction}

Deep brain stimulation is a way to stimulate parts of the brain that cause movement disorders like Parkinson's disease, in order to minimize or eliminate disease symptoms without damaging the brain. This is done by placing electrodes in specific nuclei of the brain and stimulating them with electrical impulses. Such functional neurosurgical procedures of targeting small areas deep in the brain require precise targeting. Traditionally, this is done in two steps. An approximate target location is first selected pre-operatively. The target position is then adjusted intra-operatively. Manual localization of the target is achieved pre-operatively by registering an anatomic atlas such as the Schatelbrand-Wahren atlas to a pre-operative MR scan of the patient. This step is required because the precise boundaries of structures of interest in DBS surgeries are either not or poorly visible in the pre-operative MR scans. The anatomic information afforded by the atlas is thus used as a guide. Intra-operative adjustment is based 
on microelectrode recordings (MER) obtained by passing a recording electrode in the brain towards the pre-operatively planned target and on patient's response to stimulation as a stimulating electrode moves towards the planned target. Stimulation is provided by applying electrical impulses and the responses to such stimulation include improvement in disease symptoms, occurrences of side effects, etc. Intra-operative adjustment is necessary because of known limitations of available anatomical atlases (e.g. hemispheres that pertain to different subjects, discontinuities between slices, etc.), limited accuracy achievable when registering an MR volume to these atlases, and because available atlases provide only anatomic information while the position of the final target point is chosen based on the electrophysiology. To address these issues and to simplify the procedure, we created a three dimensional electrophysiological atlas that can be used for pre-operative planning and intra-operative guidance $[2,3]$.

Over the last several years we have also developed fully automatic 3D non-rigid registration algorithms that allow us to register accurately 3D MR brain volumes to each other and we use these algorithms to develop our atlas. In the operating room, we acquire MER signals, information about stimuli (in volts), response(s) to these stimuli, and the positions at which these data are recorded. We acquire these positions in CT coordinates via the stereotactic system used for the procedure (StarFix microTargeting Platform ${ }^{\circledR}$, FHC Inc., Bowdoinham, ME). Using our registration algorithms, we then map the positions at which intra-operative information is gathered from each patient onto the corresponding positions in one MR volume chosen as a reference. This reference volume is referred to as the atlas. In this way all the intraoperative information gathered from the patients can be mapped onto the atlas for future use. The atlas thus becomes a repository that allows us to store information acquired from any number of patients in a normalized space.

Rohlfing et al. have shown that the choice of the atlas has a substantial influence on the quality of registration-based segmentation $[4,5]$. Moreover, they demonstrated that by using multiple atlases, the segmentation accuracy could be improved over that obtained using a single atlas. Here, we investigate the effect of the atlas on the accuracy of our approach to predict the position of DBS targets. We also investigate a method by which using a combination of the atlases leads to improvement in accuracy of automatic prediction.

\section{Data Set}

With IRB approval (Vanderbilt University IRB \# 010809) a set of CT and MRI preoperative scans and a CT post-operative scan is acquired for each patient. CT and MR volumes are acquired with the patient anesthetized and head taped to the table to minimize motion. Typical CT images are acquired at $\mathrm{kvp}=120 \mathrm{~V}$, exposure $=350$ mas, $512 \times 512$ pixels. In-plane resolution ranges from 0.49 to $0.62 \mathrm{~mm}$, and slice thickness from $1 \mathrm{~mm}$ to $2 \mathrm{~mm}$. MR images are 3D SPGR volumes, TR: $12.2 \mathrm{~ms}$, TE: $2.4 \mathrm{~ms}$, dimension $256 \times 256 \times 124$ voxels; voxels dimensions are typically $0.85 \times 0.85 \times 1.3 \mathrm{~mm}^{3}$. Fourteen patients who underwent STN (subthalamic nucleus) stimulation were used in the study presented herein. The patients were treated over a period ranging from December of 2003 to April of 2005. Patients included in this study are different from the ones used in our earlier work [2,3]. Therefore, although results are qualitatively similar, quantitative comparison between results presented in this work and those presented earlier is not meaningful. 


\section{Method and Results}

Four volumes were manually selected as reference volumes (atlases) based on their morphological characteristics. These volumes were not from the test set of 14 patients used in this study. They were of earlier DBS patients. They were selected to have a variety in the sizes and symmetry/asymmetry of the ventricles. Because the nuclei of interest in this study are either not or poorly visible in current image acquisition sequences, registration is driven by the surrounding structures such as the ventricles, the thalamus, or the putamen, which are visible. The size and shape of the ventricles and more particularly the size of the third ventricles were chosen as the criteria for the selection of the atlases.

\subsection{Registration Algorithms}

Two types of registrations algorithms are needed to process our data: rigid and nonrigid. The rigid registration algorithm is required to register MR and CT volumes of the same patient. The algorithm we have used for this is an independent implementation of a standard MI-based algorithm [10]. Non-rigid registration is required to register patient data to an atlas and vice-versa. In this study, non-rigid registration is always performed on MR image volumes using an algorithm we proposed recently [1].

\subsection{Influence of the Choice of the Atlas on the Prediction Accuracy}

In this work, we used the method that we described in $[2,3]$ to create an electrophysiological atlas based on the final positions of the implants. Because we now use four atlases, the process is repeated four times. Briefly, for each case we registered the MR patient volume to each the four atlases using the registration algorithms described in section 3.1. The registration parameters used for registration onto each of the four atlases were kept the same. We then projected final implant positions from each of the patients onto the four atlases, thus creating two clouds of points (one for the left and the other for the right STN) on each of the atlases. Figure 1 shows the results we have obtained for the left STN. Similar clusters were obtained for the right STN. To provide the reader with a better sense for the locations and spreads of the clusters we have superimposed contours obtained from the Schaltenbrand-Wharen (SBW) [9] atlas onto the MR images (the four atlases). Registration between each of the four atlases and the SBW atlas was performed based on a piecewise affine transformation using the Voxim software (IVS Solutions, AG, Chemnitz, Germany). This is known to be a difficult and inaccurate process. Thus, contour lines shown on the images in figure 1 may not exactly correspond to the true boundaries of the structures. The structure surrounding the core of each cluster is the STN. The structure above the STN is the thalamus with all its sub-nuclei; the structure below the STN is the substantia nigra ( $\mathrm{SNr})$.

To quantify the spread of the cluster in each atlas, we have computed the Euclidian distance from the points in each cluster to their corresponding centroid. Table 1 reports this average distance (Dc) for each of the atlases for the left and the right sides. It can be seen from table 1 that atlas0 is the best in terms of the tightness of the clusters. 

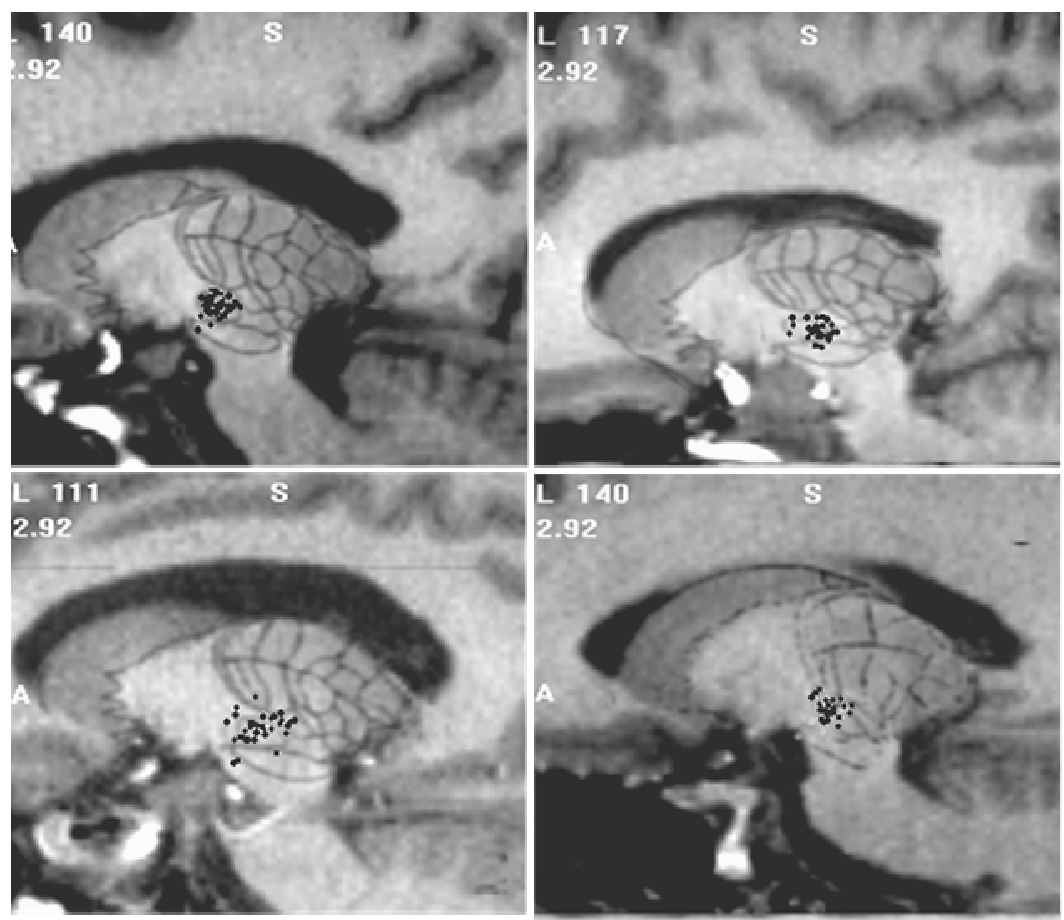

Fig. 1. Sagittal views of the right clusters on the 4 atlases (atlas 0 top left, atlas 1 bottom left, atlas 2 top right, atlas 3 bottom right). Contours extracted from the Shaltenbrand-Wahren atlas have been superimposed on the images to show the location and extent of the projected clusters with respect to anatomic structures seen in the SBW atlas.

Table 1. Euclidian distance $(\mathrm{mm})$ of cluster points with respect to the corresponding cluster centroid on each of the atlases (Dc)

\begin{tabular}{|c|c|c|c|}
\hline Atlas & $\begin{array}{c}\text { RMS spread left side } \\
(\mathrm{mm})\end{array}$ & $\begin{array}{c}\text { RMS spread right side } \\
(\mathrm{mm})\end{array}$ & Tightness ranking of atlases \\
\hline 0 & 2.01 & 2.05 & 1 \\
1 & 3.19 & 4.35 & 4 \\
2 & 3.06 & 3.11 & 3 \\
3 & 2.42 & 2.71 & 2 \\
\hline
\end{tabular}

The tightness of the cluster depends on the quality of the non-rigid registration between each of the atlases and the patient volume, which depends in large parts on the morphological similarity between the volumes being registered. A single patient volume will be registered with a higher accuracy on the most similar atlas. To study the effect of the choice of the atlas on the prediction of the optimal position for the placement of the implant we selected the centroids of the clusters in each of these 
atlases as the optimal implant positions in the corresponding atlases. These points were then projected using the transformations computed with the registration algorithms back onto each of the patients. On each of the patients this projection resulted in four possible optimal positions for the implant, each related to one of the atlases. To quantify the effect of the atlas on target prediction accuracy, we computed what we call the pre-operative placement error. This error is defined as the Euclidean distance between the final intra-operative position selected by the surgical team and the position chosen pre-operatively. It is thus the distance by which the surgical team would need to adjust the position of the electrode during the procedure. Table 2 shows the mean and standard deviation of this error for both automatic (atlas based) and manual predictions, over the 14 volumes included in this study. Columns titled Atlas0, Atlas 1, Atlas 2 and Atlas3 correspond to the distances between the automatically predicted points using the corresponding atlases and the final (intra-operative) implant position, averaged over all the patients. The column titled Centroid represents the deviation between the centroid of the four predictions and the final implant position, averaged over all the patients. The column titled Manual represents the deviation between the target predicted pre-operatively by the surgeon and the final implant position, averaged over all the patients. Table 2 shows that the choice of atlas has a direct impact on the accuracy of prediction of optimal position of implant. In earlier work, we have shown that pre-operative target points obtained automatically were closer to the corresponding final points than the pre-operative target points obtained manually. This remains true in the results shown in table 2. Moreover, what table 2 shows is that the pre-operative placement error could be reduced substantially if we could select automatically the best atlas for the case at hand, thus incurring minimum prediction error. We cannot, of course, use the pre-operative placement error as a criterion to select this atlas since the intra-operative position is not known at the time of planning. In the next section, we describe a method we propose to select the best atlas (or an optimal combination of the atlases) for a given patient.

Table 2. Prediction errors incurred for atlas based predictions using individual atlases, centroids of atlas based predictions, sensitivities of individual structures based predictions, sensitivities of combination of structures based predictions and manual selection of targets. SD: Standard Deviation, T: Thalamus, TV: Third Ventricle, P: Putamen.

\begin{tabular}{|c|c|c|c|c|c|c|c|c|c|c|c|}
\hline \multirow[t]{4}{*}{ Side } & \multirow[t]{4}{*}{ Statistic } & \multicolumn{10}{|c|}{ Deviation from final implant (mm) } \\
\hline & & \multicolumn{4}{|c|}{ Using individual atlases } & \multicolumn{5}{|c|}{ Using combination of multiple atlases } & \multirow[t]{3}{*}{ Manua } \\
\hline & & \multirow[b]{2}{*}{ Atlas0 } & \multirow[b]{2}{*}{ Atlas1 } & \multirow[b]{2}{*}{ Atlas2 } & \multirow[b]{2}{*}{ Atlas3 } & \multirow[t]{2}{*}{ Centroid } & \multicolumn{4}{|c|}{ Sensitivities weighted } & \\
\hline & & & & & & & $\mathrm{T}$ & TV & $\mathrm{P}$ & $\mathrm{T}+\mathrm{TV}$ & \\
\hline \multirow[t]{2}{*}{ Left } & Mean & 2.01 & 1.99 & 1.72 & 1.9 & 1.8 & 1.8 & 1.71 & 1.93 & 1.66 & 2.23 \\
\hline & SD & 0.85 & 0.94 & 1.11 & 0.99 & 0.93 & 0.93 & 0.89 & 0.85 & 0.92 & 1.17 \\
\hline \multirow[t]{2}{*}{ Right } & Mean & 1.92 & 2.21 & 2.13 & 2.09 & 1.97 & 1.87 & 1.98 & 2.34 & 1.74 & 2.5 \\
\hline & SD & 0.69 & 1 & 0.97 & 0.99 & 0.83 & 0.8 & 0.87 & 0.85 & 0.74 & 1.41 \\
\hline
\end{tabular}




\subsection{Multiple Atlases Based Prediction}

The easiest way to combine the predictions is to compute the centroid of the four predicted points and use it as the best automatic prediction. Prediction accuracy using the centroid of the cluster is substantially better than that based on manual predictions as can be seen in table 2. However, it is still not as good as that achievable using the best atlas for every patient. For instance, it was found that for the left side, using the best atlas for each of the 14 patients the pre-operative error was $1.46 \mathrm{~mm}$ averaged over the patients, while the same based on the centroid of the cluster was $1.8 \mathrm{~mm}$ and that based on manual predictions was $2.23 \mathrm{~mm}$. To select one atlas (or perhaps a subset of atlases) for a particular case, we need to find a way to estimate the likelihood that the atlas is more accurately registered to the patient than any other atlas. We achieve this through atlas-based segmentation of structures surrounding the target of interest. As discussed above, the STN is poorly visible in MR images. But surrounding structures such as the ventricles, the thalamus and/or the putamen can bee seen and have relatively well defined boundaries. To take advantage of this, the major basal ganglia structures (the putamen, the thalamus, the ventricles, the third ventricle, the red nuclei and the globus pallidus) were manually segmented on each of the four reference volumes by an expert. These segmented structures were projected from the four atlases onto the patients, resulting in four different segmentations for each structure on every patient. These contours were then used to estimate the specificity and sensitivity of each of the four segmentations for every structure using the STAPLE algorithm proposed by Warfield et al. [7, 8]. The computed sensitivity parameters were used, in turn, to weigh the contributions of the atlases to the prediction. We used a simple weighted average of the predictions made by the four atlases to arrive at the final automatic prediction.

The weights are the sensitivities of the segmented structures for the four atlases. Sensitivities below $95 \%$ of the highest sensitivity were set to zero. Let $P 0, P 1, P 2$ and $P 3$ be the predictions based on atlases $0,1,2$ and 3 respectively and sensitivity be denoted by $p$. Let the sensitivity for the left thalamus of the patient with respect to atlas 0 be $p \_t h a l \_l e f t \_0$, that for the right thalamus with respect to atlas0 be p_thal_right_O and that for the third ventricles with respect to atlas0 be p_third_ventricles_0. A similar nomenclature for sensitivities with respect to atlases 1,2 and 3 is used. Now, the average sensitivity of the structures on the patient volume with respect to atlas 0 is given by

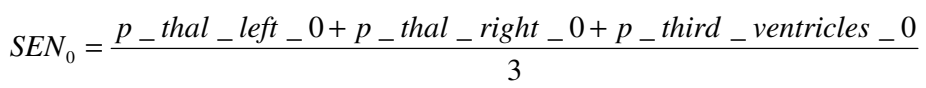

Similarly, $S E N_{1}, S E N_{2}$ and $S E N_{3}$ are computed. Next we define the set of indices IND such as

$$
\mathrm{IND}=\left\{\mathrm{k} \mid \mathrm{SEN}_{\mathrm{k}} \geq 0.95 * \max \left(\mathrm{SEN}_{0}, \mathrm{SEN}_{1}, \mathrm{SEN}_{2}, \mathrm{SEN}_{3}\right)\right\} .
$$

Then, the final prediction is given by,

$$
P=\frac{\sum_{k \in I N D} S E N_{k} \cdot P_{k}}{\sum_{i \in I N D} S E N_{i}}
$$


In doing this, we eliminate the contributions of atlases that produce low sensitivity values for the structures, i.e., atlases that lead to poor segmentation results for structures surrounding the structure of interest. Table 2 also shows the prediction error when the sensitivities of the thalamus, the third ventricle, and the putamen are individually used. These results show that, though predictions based on sensitivities of a surrounding structure are better than manual predictions, the use of sensitivities of the putamen has shown to increase the error compared to the use of the centroid. Similarly, it can be seen that the use of sensitivities of the thalamus improves the accuracy of prediction for the right side, while the use of third ventricles improves the accuracy of prediction for the left side, both compared to the use of centroid. This suggests that combining the sensitivities of the thalamus and of the third ventricles could further improve the results as corroborated in table 2 .

\section{Discussion and Conclusion}

From table 2, it can be seen that, using the sensitivities of the thalamus and the third ventricles together, the pre-operative error for the left side decreased to $1.66 \mathrm{~mm}$ compared to $1.8 \mathrm{~mm}$ when the centroid of the cluster was used and $2.23 \mathrm{~mm}$ when manual prediction was done. Similarly, for the right side, the errors were $1.74 \mathrm{~mm}$, $1.97 \mathrm{~mm}$ and $2.5 \mathrm{~mm}$ respectively. These results are clinically important because they could improve the quality of DBS surgeries both in terms of accuracy and time.

The quality of segmentations achieved on the patient volume based on each of the atlases is a direct indication of the accuracy with which each of the corresponding atlases has been non-rigidly registered to the patient volume. Since the segmentations used are from regions around the (poor contrast) STN, our method serves as an approach to assessing the quality of non-rigid registrations in regions of low contrast in a patient volume. In our multi-atlas approach to prediction of optimal points for implants in DBS surgeries, we use the above method of assessing the quality of registrations to determine the contributions of the four atlases to the prediction process. It is conclusive from our results that the use of multiple atlases helps improve the accuracy of automatically predicting optimal positions for DBS implants. This scheme can easily be extended to other targets by changing the visible structures used to evaluate the quality of the registration. For instance, in the prediction of the target points for Globus Pallidus Internus (GPi), the putamen can be expected to play a key role due to its close proximity to GPi.

\section{Acknowledgments}

Pierre-François D'Haese is working towards a Ph.D. degree with awards from FRIA and FNRS (Belgian Science Foundation) (also supported by the Walloon Region by the MERCATOR grant). Part of this work has been supported by a Discovery grant from Vanderbilt University. 


\section{References}

1. Rohde G.K., Aldroubi A., Dawant B.M., "The adaptive bases algorithm for intensity based non-rigid image registration." IEEE Trans Med Imaging, 2003 Nov; 22(11): 1470-9.

2. Dawant B.M., Li R., Cetinkaya E., Kao C., Fitzpatrick J.M., and Konrad P.E., "Computerized Atlas-Guided Positioning of Deep Brain Stimulators: A Feasibility Study", J.C. Gee et al. (Eds.): WBIR 2003, LNCS 2717, pp. $142-150,2003$.

3. D'Haese P.F., Cetinkaya E., Kao C. , Konrad P.E. and Dawant B.M. "Computer-aided Placement of Deep Brain Stimulators: From Planning to Intra-operative Guidance” IEEE Trans Med Imaging, Special Issue on Computer Aided Surgery, 2005 (In Press).

4. T.Rohlfing, C. Maurer, Jr., "Multi-Classifier framework for atlas-based image segmentation", Proceedings of IEEE Computer Society Conference on Computer Vision and Pattern Recognition, Washington D.C., USA, June 27-July 2, 2004, pp. 255-260, IEEE Press, 2004.

5. T.Rohlfing, D. B. Russakoff, C. Maurer, Jr., "Performance-based classifier combination in atlas-based image segmentation using Expectation-Maximization parameter estimation", IEEE Transactions on Medical Imaging, vol. 23, pp. 983-994, 2004.

6. Ebru Cetinkaya, Pierre-Francois D'Haese, Peter E. Konrad, Chris Kao, and Benoit Dawant. "Method for identifying brain nuclei from micro-electrode signals". ASSFN 2004, Cleveland, USA, Oct', 2004.

7. S. K. Warfield, K. H. Zou, W. M. Wells, "Validation of image segmentation and expert quality with an Expectation-Maximization algorithm", Lecture Notes in Computer Science, MICCAI 2002, pp. 298-306, 2002.

8. S. K. Warfield, K. H. Zou, W. M. Wells, "Simultaneous Truth and Performance Level Estimation (STAPLE): An algorithm for the validation of image segmentation", IEEE Transactions on Medical Imaging, vol. 23, no. 7, pp. 903-921, 2004.

9. G. Schaltenbrand and W. Wahren, "Atlas for Stereotaxy of the Human Brain" Stuttgart, Germany; Thieme, 1977.

10. Li R., "Automatic placement of regions of interest in medical images using medical image registration”, Master's thesis, Vanderbilt University, Dec' 2001. 